Thorax (1960), 15, 70.

\title{
SOME FEATURES OF THE MITRAL VALVE
}

\section{BY}

\author{
E. W. T. MORRIS
}

From the Department of Anatomy, St. Thomas's Hospital Medical School, London

(RECEIVED FOR PUBLICATION OCTOBER 28, 1959)

Examination of a series of fresh normal hearts has revealed certain features of the anatomy of the mitral valve that are not mentioned in textbooks of anatomy or in clinical articles.

Briefly, the fibrous ring to which the cusps of the mitral valve are attached does not lie in one plane as shown in standard textbooks, and consequently the relationship of the anterior, aortic, or septal cusp of the valve to the left atrium, and to the inflow and outflow channels of the left ventricle, is not that which would be inferred if the ring were in one plane.

The fibrous ring, part of the framework of the heart, which separates atrium from ventricle, and to which the cusps of the mitral valve are attached, $\vec{x}$ consists, in its superomedial part, of the borders $\vec{T}$ of the right and left fibrous trigones, and the fibrous wall of the subaortic part of the left ventri- $\triangle$ cular chamber with which they are continuous.을 The remaining and inferolateral part of the ringis continuous with the trigones (Fig. 3). The ring so formed does not lie in one plane but is hinged about an axis which joins the two commissures ${ }^{-}$ of the valve cusps (Figs. 1 and 3).

The shape of the anterior cusp can be seen in: Fig. 4. Its attached border forms an arc, ando its free border is subdivided into three regions: a middle one the centre of which is free from
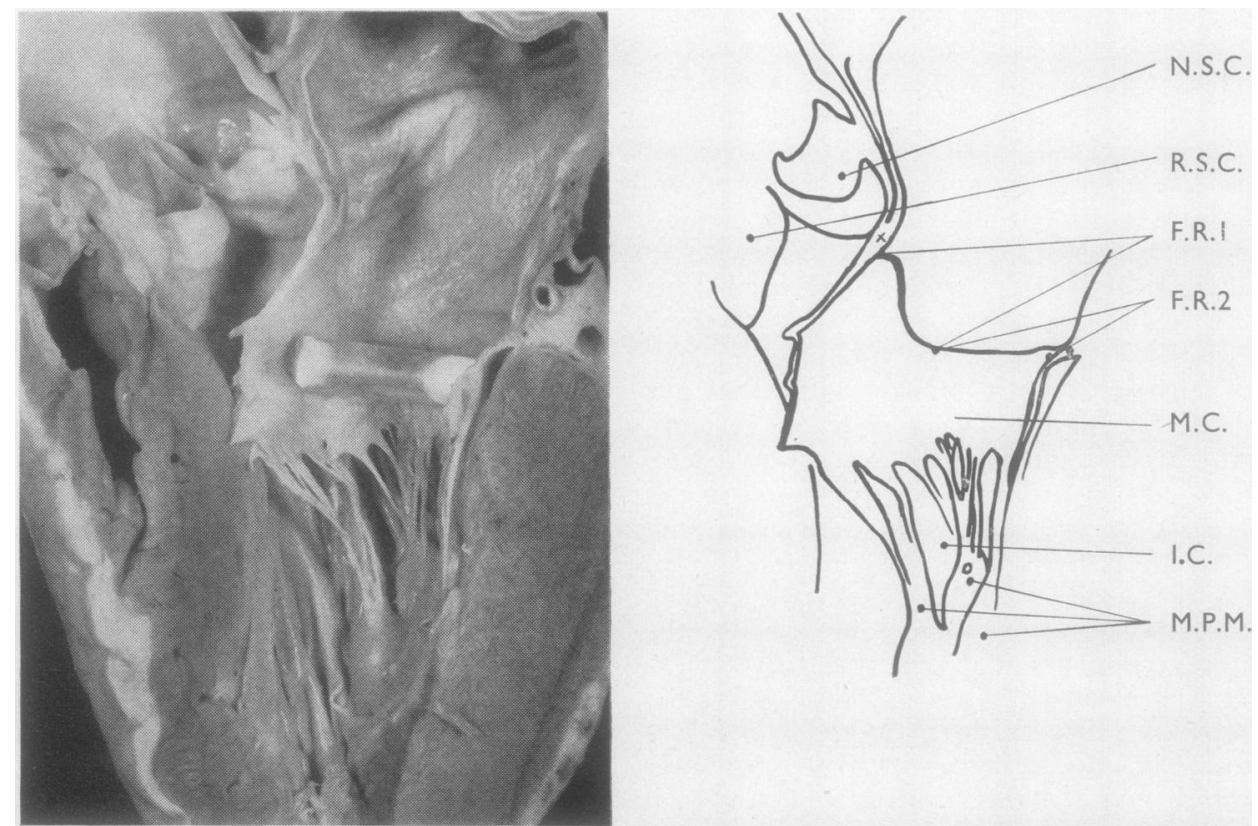

FIG. 1.-The right half of the aorta and left atrium and ventricle divided down the centre of the anterior cusp of the mitral valve which has been propped open to represent its position in diastole. The junction of anterior cusp and fibrous ring is, marked $X$.

A., Aortic wall. A.C., Anterior cusp of mitral valve. A.M., Wall of left atrium. B., Junction of A.C., A.M., and I.V.S. C., Cusp of aortic valve. F.R.1, Anteromedial part of the fibrous ring to which anterior cusp is attached. F.R.2, Posterolateral part to which posterior cusp is attached. I.C., Inflow channel. I.V.S., Intervalvular space between left septal and nonseptal cusps of the aortic valve. L.T., Left fibrous trigone. M.C., Medial commissure. M.P.M. Medial papillary muscle. N.S.C., Nonseptal cusp of the aortic valve. O.C., Outflow channel. R.S.C., Right septal cusp Medial papillary muscle. N.S.C., Nonseptal cusp of the aortic valve. O.C., Outflow cha
of the aortic valve. R.T., Right fibrous trigone. 
chordae tendineae, and two lateral regions which recede from it towards the fibrous ring. The contour of the free border is responsible for the

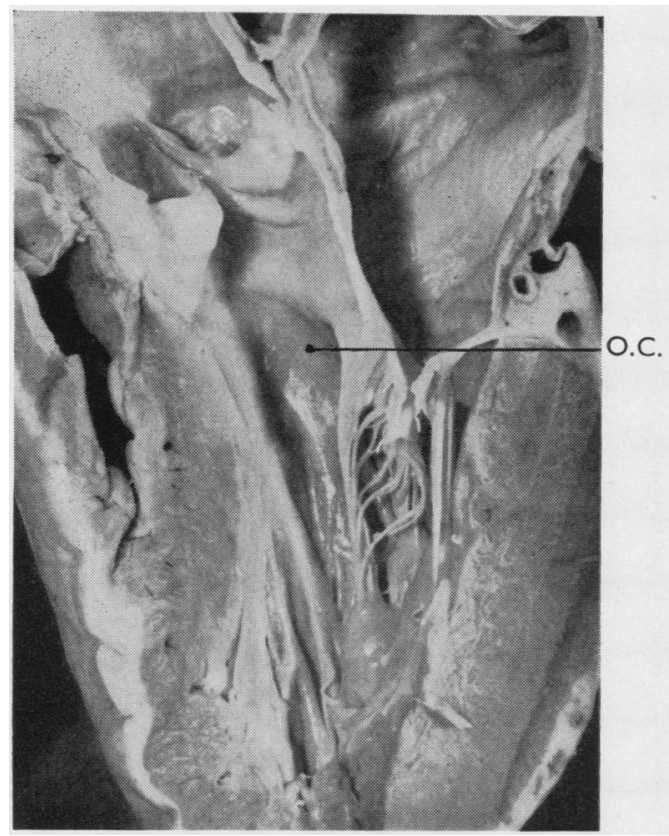

FIG. 2.-Same as Fig. 1 with the cusps approximated. The intervention of the anterior cusp between the atrium and the subaortic part of the outflow channel is shown.
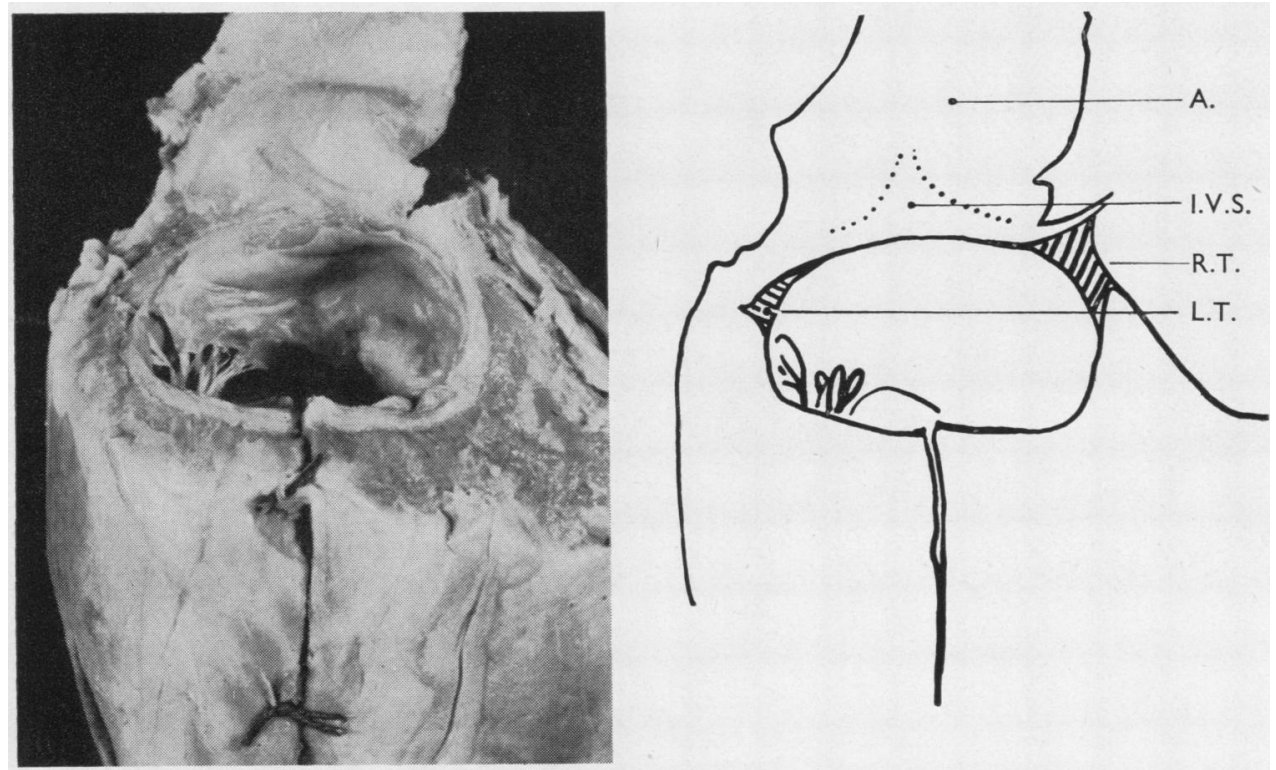

FIG. 3. The left heart seen from behind. The atrium has been removed to show the fibrous ring and trigones. The sites of the left septal and nonseptal cusps in the aorta are indicated. characteristic crescentic form of the closed aperture. The centre of this cusp is situated immediately below the intervalvular space between the left septal and nonseptal cusp of the aortic valve (Figs. 3, 5, and 6).

The cusp contains a well-developed sheet of fibrous tissue, the lamina fibrosa, continuous at its attached border with the fibrous framework of the heart (Fig. 6). It does not extend quite to the free border. The chordae tendineae insert into the lamina fibrosa. The posterior (inferior) cusp is similar in structure to the anterior, but is narrower and more rectangular.

The greatest width of the cusps from free to attached borders was measured in 16 consecutive

TABLE

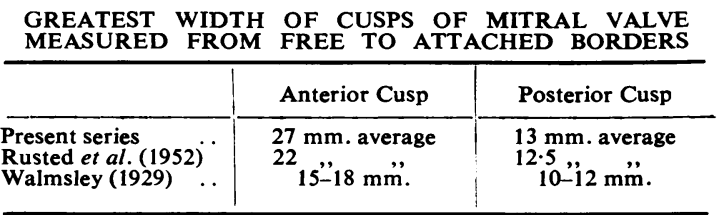

fresh hearts. The width of the anterior cusp was found to be greater than that recorded by others, but the measurement of the posterior cusp corresponded quite well (see Table).

At neither end did the aperture between the cusps extend to the fibrous ring in any of the hearts examined. There was always a commissure 


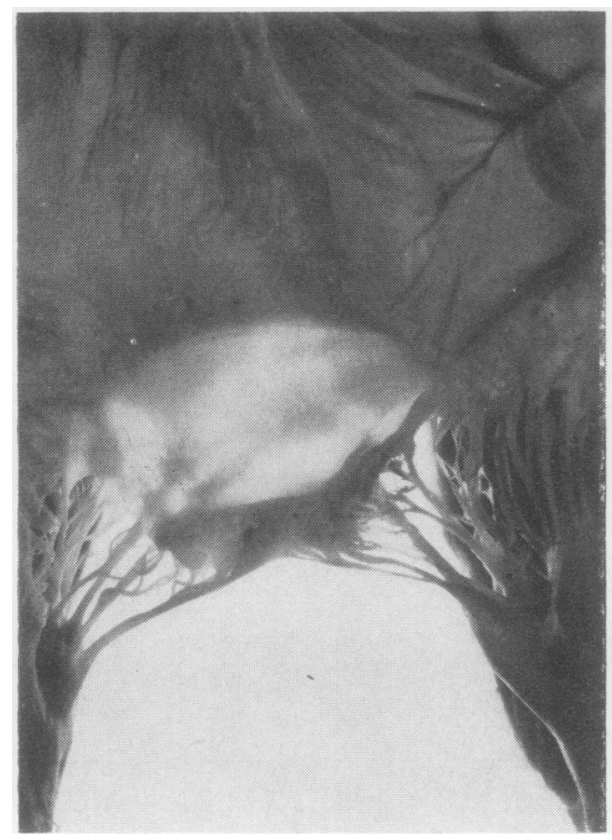

FIG. 4.-The anterior cusp seen from the atrial side.

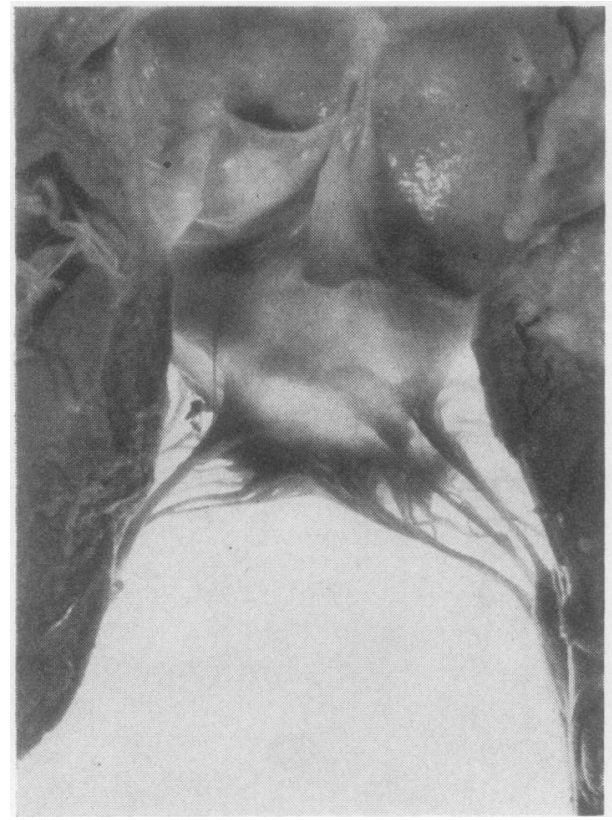

Fig. 5.-The anterior cusp from the ventricular aspect. The left septal cusp of the aortic valve overhangs and obscures the intervalvular space.

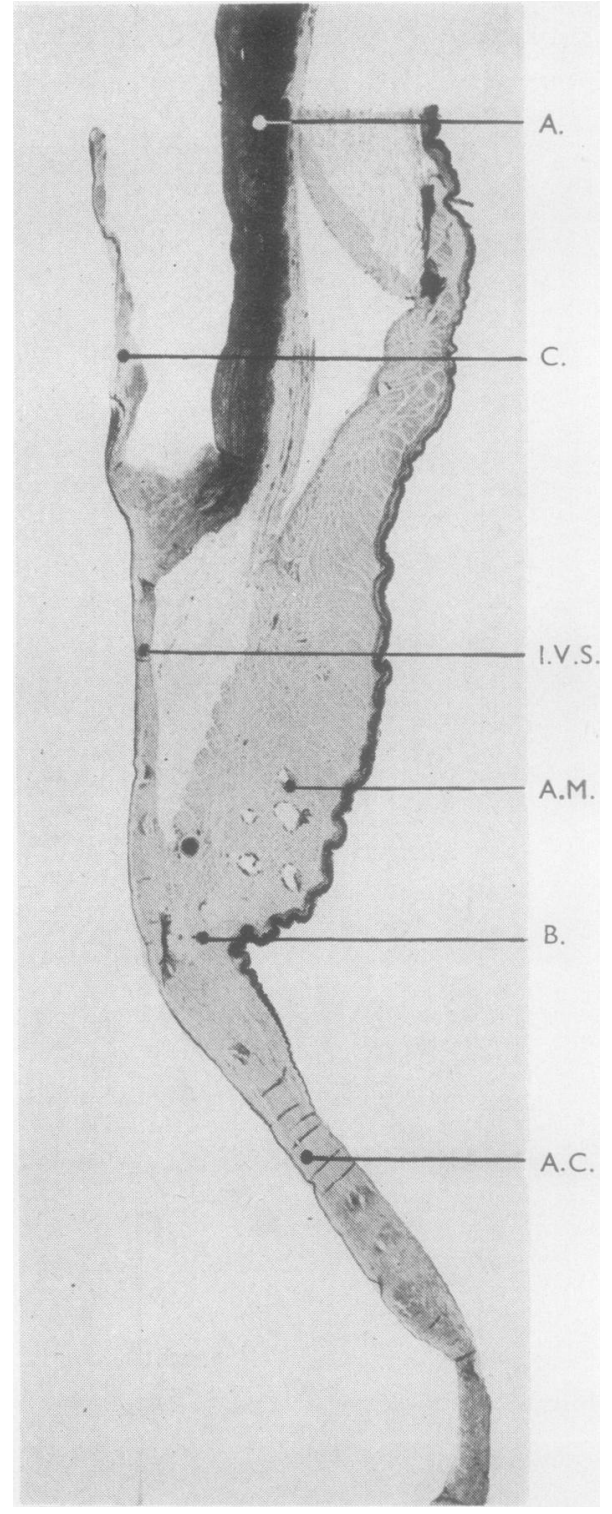

Fig. 6.-A section from near the middle of the anterior cusp of the $\boldsymbol{\omega}$ mitral valve and adjacent structures stained with orsein, $\times 5$.

several millimetres in length along which the tissue of adjacent cusps was continuous. Notches are $\stackrel{\mathcal{Q}}{\rightarrow}$ commonly seen in the free border of the posterior 0 cusp, especially when the valve is shut. These assist in adapting the free border of the posterior $\stackrel{\mathbb{D}}{\Omega}$ cusp to the three subdivisions of the free border $\underset{\mathbb{D}}{\mathbb{Q}}$ of the anterior cusp.

The cavity of the left ventricle is divided into an inflow and an outflow channel. The formero 
is directly anterior to the atrioventricular orifice (Fig. 1) and the latter above and medial to it (Fig. 2).

The inferior wall of the inflow channel is the proximal part of the inferior wall of the ventricle as far as the attachments of the papillary muscles. Its superior wall is the anterior cusp of the mitral valve, the lower border of which forms the upper boundary of the communication between the two channels. The remainder of the boundary is formed by the superior borders of both papillary muscles and the chordae tendineae connecting them to the aortic cusp. A further restricted communication exists between the same parts of the papillary muscles and the adjacent ventricular wall.

In the course of ventricular systole, first the inflow, then the outflow channels are obliterated by coaption of the walls and papillary muscles leaving finally a space immediately below the aortic valve which is persistently patent (Fig. 1).

The disposition of the fibrous ring in relation to the atrium and ventricle is such that, while the lower part of the anterior cusp separates inflow and outflow channels, its superior part separates the persistently patent region of the outflow channel from the left atrium. Such a relationship would make any breach of this barrier a serious embarrassment to function.*

I wish to express my thanks to Miss E. Petley and Mr. A. L. Wooding for the photographs.

\section{REFERENCES}

Rusted, I. E., Scheifley, C. H., and Edwards, J. E. (1952). Circulation, 6,825 .

Sibson, F. (1869). Medical Anatomy. Churchill, London.

Walmsley, T. (1929). Ouain's Elements of Anatomy, 11th ed. Ed. T. H. Bryce and T. Walmsley, Vol. IV, Pt. 3. The Heart. Longmans, Green, London.

* Just as this paper was completed the writer discovered an excellent description of the mitral valve and left ventricle in Sibson (1869) which confirms most of the features set out above. 